\title{
Study on the Effects of Grinding Factor and Scaling up in Grinding Using Horizontal Dry Bead Mill
}

\author{
Takahiro TAMURA $^{1 *}$ and Mitsumasa KIMATA ${ }^{2}$ \\ ${ }^{1}$ Institute of Fine Particle Technology from Ashizawa Finetech Ltd., 2-1-4 Hitotonoya, Oyama, Tochigi 323-0034, Japan \\ ${ }^{2}$ Department of Chemistry and Chemical Engineering, Yamagata University, 4-3-16 Jonan, Yonezawa, Yamagata 992-8510, Japan
}

\begin{abstract}
Effect of grinding factor and scaling up were carried out by grinding using horizontal dry bead mill. For the experiments, 2 sizes of horizontal dry bead mills with different capacities, lab and manufacturing scale, were used to study scaling up and down. The grinding experiments were performed by changing each typical grinding factor for bead mills, including the agitator peripheral speed, bead packing density, bead diameter, and material feeding speed. This set of experiments was conducted for 3 kinds of materials, silica sand of natural inorganic materials, alumina of synthetic inorganic materials, and activated carbon of organic materials. As a result, common for all materials, similar rules were found between the particle size and specific energy, the amount of mechanical energy per unit amount of material. In addition, these rules were applicable for the different-size mills, indicating that the scale can be controlled by the rules and specific energy. Also, it is revealed that when using a single type of material, changing the bead diameter changes the energy efficiency, indicating that using small-diameter bead was effective for efficient grinding.
\end{abstract}

\section{Introduction}

Although milling operation had been a well-known unit operation, it often held a prominent position in the industry up until today. Therefore, number of types of grinder had been developed to fulfil the demands of the times (Matsuyama, 1959a, 1959b; Tanaka, 1965, 1979). The industry focuses on time and energy needed for a grinding machine to pulverize a certain amount of milling material, that is, time efficiency and energy efficiency of the machine, and these are significant elements for evaluating the performance of a grinding machine. Interest in energy efficiency will further increase especially in today's industry, where awareness of environmental loading reduction is increasing while subdivided powder sizes which requires larger energy is demanded. On the other hand, as wide variety of products being produced, milling material has been expanding its range to handle from conventional products to high-valueadded products, and its production style has been diversified from mass production to large item small scale production.

With the ability to provide high density filling of medium and high energy density milling environment, and the ability to handle a variety of milling operation in principle, bead mill is greatly expected to be used to fulfil today's industry's needs which demands various milling operation. Because, bead mills can provide grinding conditions under a high packing density and high energy density of the media as well as cover a broad range of output (Tanaka, 1994). However, because of its short history, it is hard to say that, especially dry bead mill, is well known by general public. Here, we have decided to examine and report the performance of horizontal dry bead mill.

\section{Experimental}

\subsection{Test equipment}

The outline and the specification of the test equipment used for this experiment are shown in Figure 1 and Table 1 , and the experimental condition is shown in Table 2 . The equipment was operated with contentious path operating method with constant feeder on dry bead mill. Two types of bead mill of different capacities, laboratory scale (Ashizawa Finetech Ltd., DRYSTAR SDA1), and production scale (Ashizawa Finetech Ltd., DRYSTAR SDA5), were used to examine scale up/down. Screw feeder (KUMA Engineering Co., Ltd., Accurate Feeder302) was used for constant feeder. The milling material (feed particle) used for this grinding equipment was fed into the milling chamber with milling aid which is added from milling aid charging opening before being fed into the milling chamber via constant feeder. Then, pulverizing was done in multiple stages by rotating the agitator in the milling chamber to apply movement to beads, which contact with milling materials, and then the milling materials and beads were separated by a screen installed near the outlet to be extracted as powder composition.

\footnotetext{
* Corresponding author: t-tamura@ashizawa.com
} 


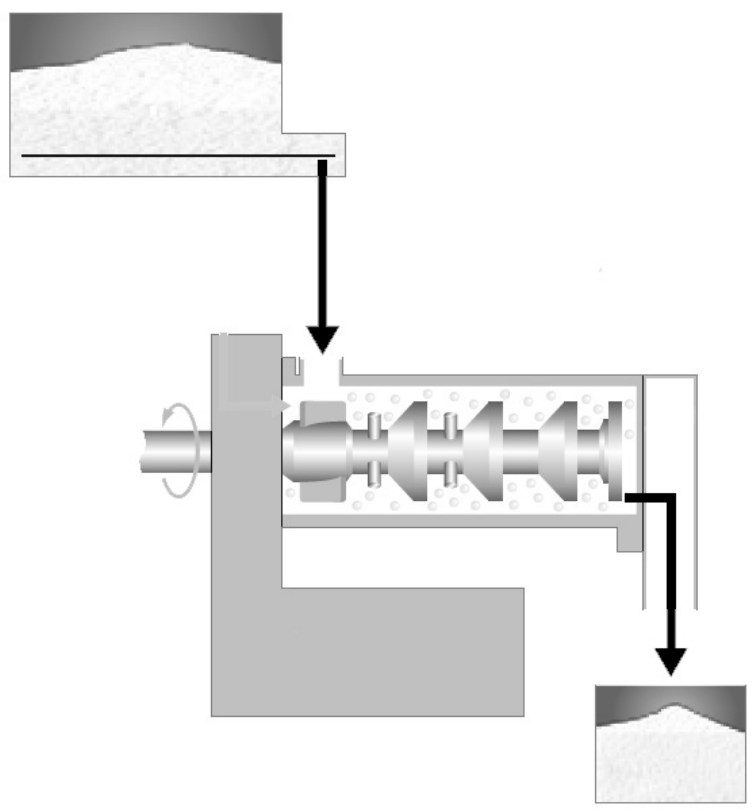

Figure 1. Machine unit of dry grinding

Table 1. Machine conditions

\begin{tabular}{|c|c|}
\hline Machine & SDA5 \\
\hline Vessel volume & $4.0 \mathrm{dm}^{3}$ \\
\hline Vessel material & Stainless steel (SUS304) \\
\hline Agitator material & Tool steel (SKD) \\
\hline Beads material & $\begin{array}{l}\text { Partially stabilized zirconium oxide } \\
\text { (PSZ) }\end{array}$ \\
\hline Machine & SDA1 \\
\hline Vessel volume & $1.0 \mathrm{dm}^{3}$ \\
\hline Vessel material & Stainless steel (SUS304) \\
\hline Agitator material & Stainless steel (SUS304) \\
\hline Beads material & $\begin{array}{l}\text { Partially stabilized zirconium oxide } \\
\text { (PSZ) }\end{array}$ \\
\hline
\end{tabular}

Table 2. Grinding conditions

\begin{tabular}{|c|c|c|c|}
\hline Material & Silica sand & Almina & Carbon \\
\hline $\begin{array}{l}\text { Agitator peripheral } \\
\text { speed }[\mathrm{m} / \mathrm{s}]\end{array}$ & $3-6$ & $4-5$ & $4-5$ \\
\hline Bead diameter $[\mathrm{mm}]$ & $1.5,3,5$ & 3 & 3 \\
\hline $\begin{array}{l}\text { Filling rate of beads } \\
\text { [vol\%] }\end{array}$ & $65-80$ & $70-80$ & 80 \\
\hline Feed $[\mathrm{kg} / \mathrm{h}]$ & $0.4-5.5$ & $2.5-15$ & $1.0-5.0$ \\
\hline $\begin{array}{l}\text { Ration of grinding aid } \\
\text { in products[mass } \%]\end{array}$ & 1.0 & 1.0 & 0 \\
\hline
\end{tabular}

\subsection{Experiment condition}

Pulverizing experiment was implemented by changing each operation variables such as circumferential speed of agitator, packaging ratio of the bead, bead size, and supply rate of milling material, which are considered to be typical on using the test machine. The experiment was implemented on three different milling materials: silica sand, a natural inorganic substance, alumina, inorganic composition, and active carbon, an organic substance.
During pulverizing silica sand and alumina, ethyl alcohol and propylene glycol was used respectively as milling aid to prevent the adhesion of powder during pulverizing (Hasegawa et al., 2001, 2002) and to operate safely. Powder composition extracted from the grinding machine was measured with lead cell, and power required for milling was obtained from dynamometer accompanying the grinding machine. Also, the particle size of milling material and powder composition was measured using laser diffraction particle diameter distribution measuring machine (Micro-Trak MT3000).

\section{Results and Discussion}

\subsection{Relationship between specific energy and particle size}

Each milling condition used in this research (circumferential speed of agitator, packaging ratio of the bead, bead size, and supply rate of milling material) can be a factor to change the property inside the grinding machine, and therefore, it is considered to have influence on the mechanical power of the grinding machine. We considered full power of the machine during pulverizing as $E_{t}[\mathrm{~kW}$, and air power at the same circumferential speed as $E_{n}[\mathrm{~kW}]$, and showed the actual power $E_{f}[\mathrm{~kW}]$ in Eq. (1), then normalized this value using the supply rate of milling material $W$ and considered this as specific energy $S_{n}[\mathrm{~kJ} / \mathrm{g}]$.

$$
S_{n}=\frac{\left(E_{t}-E_{n}\right)}{W} \times 3.6=\frac{E_{f}}{W} \times 3.6
$$

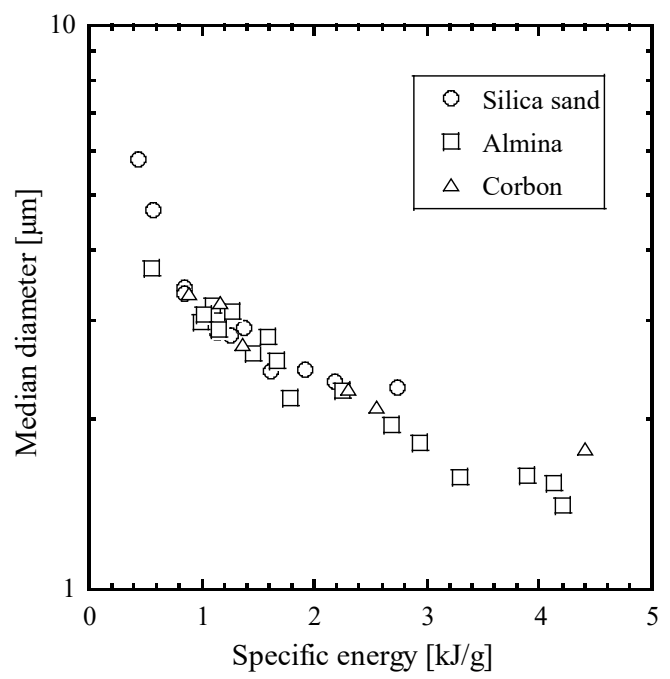

Figure 2. Relationship between the specific energy and the particle size in each material

Figure 2 shows the relationship between specific energy and particle size, where $\varphi 3 \mathrm{~mm}$ bead was used for pulverization of each milling material. Here, median diameter $\left(D_{50}\right)$ of the standard value was used as a representative value of particle size. The diagram shows that for pulverization of each milling material, there is a 
correlation between specific energy and particle size. Moreover, since specific energy is associated with actual power, which is affected by key parameters of dry bead mill, and the supply rate of milling material, it should be possible to control particle size and the production volume of powder by adjusting specific energy.

\subsection{Specific energy and scale controlling of grinding machine}

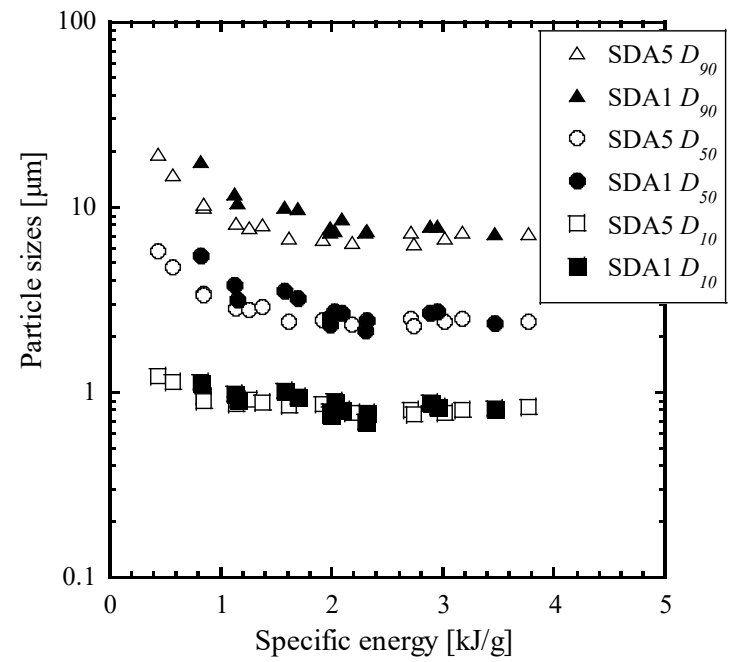

Figure 3. Relationship between the specific energy and particle sizes which were used $D_{50}, D_{10}$, and $D_{90}$ by using SDA5 and SDA1.

Due to mechanical restrictions of set range of actual power and supply rate of milling material, there is also a restriction of production volume. Therefore, if there are demand to boost the output of production, either scale-up, where machine size is increased, or numbering-up, where the number of machines is increased, are the typical measures to be taken to meet such demand. Although numbering-up can assure the increase in the output of production volume of the same powder, it consumes more installation area of machine tools since the number of machines installed increases. While scale-up can be implemented without consuming installation area of machine tools, the difficulty to determine the condition to obtain the same powder with different scales can be a disadvantage. However, if it is possible to define the selection criteria of conditions, scale-up can be an extremely reasonable way to boost the output of production. Here, Figure 3 shows the test result of pulverizing silica sand using dry bead mill that was used in this experiment with different capacities, expressed by using specific energy and particle sizes which were used $D_{10}, D_{50}$, and $D_{90}$. Bead size we used here was also $\phi 3 \mathrm{~mm}$. The diagram shows that the relationship between specific energy and particle size is maintained regardless of the size of the machine, indicating that upon scaling up dry bead mill, pulverizing conditions for scale-up, motor capacity of the grinding machine, and milling material supply feeder can be determined based on specific energy needed to obtain the specified particle size. Further, it has been reported by Ishii et al. $(2011,2012)$ that the relationship with particle size can be controlled by specific energy when bead mill in liquid phase (wet grinding) is used. Combined with the result of this experiment, pulverizing process can also be optimized in consideration of combining wet and dry bead mill.

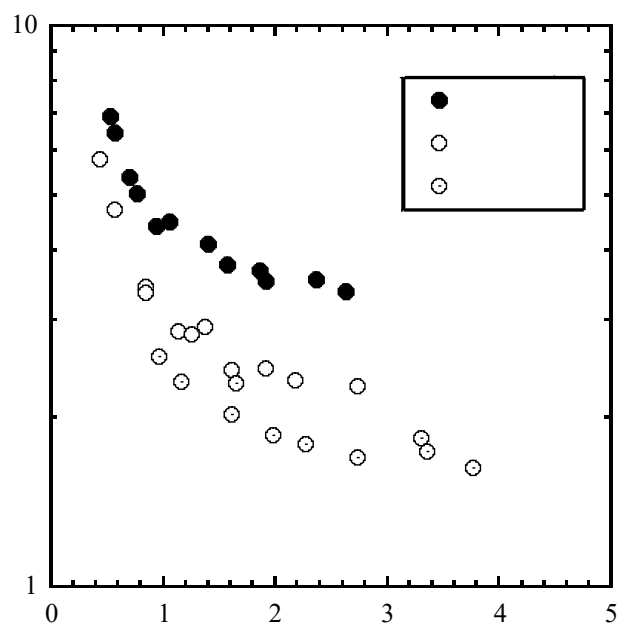

Figure 4. Relationship between the specific energy and particle size in each bead sizes.

\subsection{Energy efficiency of pulverization and using small diameter bead}

Since pulverization is an important unit operation in the industry, massive energy is being used for this process throughout the world. However, it has been said that out of energy consumed for pulverization, only less than $1 \%$ is actually used for pulverization and the rest turns into heat (Jimbo, 1971). In addition, Figure 2 shows that in each milling material, pulverization process gets harder as particle sizes turn smaller, indicating smaller the particle size, lower the energy efficiency for pulverization. Although it is believed that this results from combined action of influence of dimension effect (Sikong et al., 1989) or reduction of pulverization probability inside the grinding machine (Kanda et al., 1989) due to milling material, as mentioned at the beginning, in a modern industry where variety of particle have to be handled, it is important to increase energy efficiency of pulverization. One of the measures to increase energy efficiency of pulverization with dry bead mill is the use of small diameter bead. The reason why pulverization frequency can be drastically improved by using small diameter bead, is because contact frequency with milling material increases and higher energy will be applied to the bead from agitator because of the principle of bead mill. Also, Kanda et al. (1989) and Tanaka (1994) proposes the benefits of using small diameter bead from different view points. Therefore, we conducted a pulverization test of silica sand using beads with different diameters. Figure 4 shows the relationship between specific energy used for pulverization and median diameter. The diagram demonstrates that the smaller bead diameter requires smaller specific energy for pulverization, showing that 
energy efficiency of pulverization increases by using small diameter beads. Also, as the experiment showed that each bead diameter has pulverization limit, where pulverization stops (Tanaka, 1954), the diagram shows the limit particle diameter of each bead diameter. Although the reason why pulverization limit exists has to be examined, the experiment showed that bead diameter is a parameter which increases energy efficiency of dry bead mill, and a parameter to fulfill pulverization in finer range.

\section{Conclusion}

Each of the main parameters used for investigation on pulverization using dry bead mill, which are circumferential speed of agitator, packaging ratio of the bead, bead size, and supply rate of milling material, are considered to influence the specific energy, which is the mechanical work volume per milling material unit quantity, and the experiment showed that by arranging them, there is a correlation between specific energy and median diameter when pulverizing any milling material. In addition, this relationship was maintained during pulverization test using machine with different sizes, indicating that specific energy can be utilized as an indicator to regulate size and production volume of powder, including scale-up. In addition, the experiment showed that if the same milling material is being used, energy efficiency can be improved by changing bead diameter to be used, and using small diameter bead can be effective to execute effective pulverization.

\section{References}

Hasegawa, M., M. Kimata, M. Shimane, T. Shoji, and M. Tsuruta; "The Effect of liquid Additives on Dry Ultrafine Grinding of Quartz," Powder Technol., 114, 145-151 (2001)

Hasegawa, M., M. Kimata, and T. Shoji; "Effect of Liquid Additives and Behavior of Alumina Powder in Ultrafine Grinding Of Alumina," J. Soc. Powder Technol., Japan, 39, 736-742 (2002)

Ishii, T. and K. Hashimoto; "The Effect of Grinding Conditions on Particle Size of the Ground Particle Obtained," J. Jpn. Soc. Colour Mater., 84, 163-168 (2011)

Ishii, T. and K. Hashimoto; "Evaluation of Grinding Characteristics of Calcium Carbonate by Horizontal Bead mill," J. Jpn. Soc. Colour Mater., 85, 53-58 (2012)

Jimbo, G.; "Grinding Operation and Energy (in Japanese)," Kagaku Kogaku, 35, 286-289 (1971)

Kanda, Y. and S. Yashima; "A Consideration of Ultrafine Grinding Based on Fracture Mechanics Single Particle Crushing," Bull. Res. Inst. Miner. Dressin. Metall, 45, 3038 (1989)
Matsuyama, T.; "Progress of Powder Technology (in Japanese)," Kagaku Kogaku, 23, 705-709 (1959a)

Matsuyama, T.; "Machin Selection of Mills (in Japanese)," Kagaku Kogaku, 23, 723-727 (1959b)

Sikong, L., H. Hashimoto, and S. Yashima; "Size Effect of Breakage Properties of Fine Single Particles," Bull. Res. Inst. Miner. Dressin. Metall, 45, 23-29 (1989)

Tanaka, T.; "A New Concept Applying a Final Fineness Value to Grinding Mechanism," Kagaku Kogaku, 18, 160-171 (1954)

Tanaka, T.; "About Grinding Operation (in Japanese)," Kagaku Kogaku, 29, 650-655 (1965)

Tanaka, T.; "Recent Techniques and Theories of Comminution," Shikizai, 52, 486-493 (1979)

Tanaka, T.; "Determining the Optimum Diameter of Grinding Media Used for Ultrafine Grinding Mechanisms," J. Soc. Powder Technol., 31, 25-31 (1994) 